332 Henrich u. Eisenach: Einw. von salp. Säure etc.

\title{
Über die Einwirkung von salpetriger Säure auf Resorcinmonomethyläther;
}

vou

Ferd. Henrich und H. Eisenach.

In Gemeinschaft mit O. Rhodius hat der eine von uns vor zwei Jahren die Einwirkung von salpetriger Säure auf Resorcinmonomethyläther untersucht. ${ }^{2}$ ) Wir gaben an, drei Mononitrosoderivate erhalten zu haben, denen die Formeln:
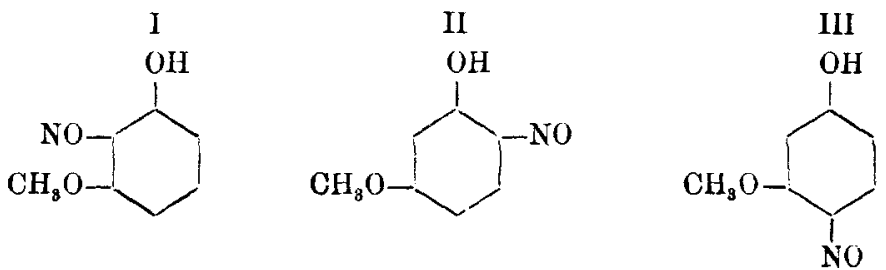

zukommen mußten. Damals konnte nur eine Verbindung ihrer Konstitution nach bestimmt werden und zwar die, welche die Nitroso- und Hydroxylgruppe in Parastellung enthielt, der also die Formel III zukam. Für die anderen Nitrosokörper blieb noch die Auswahl zwischen den Formeln I und II.

Den obigen drei Nitrosoderivaten entsprechen drei Reduk. tionsprodukte, nämlich:

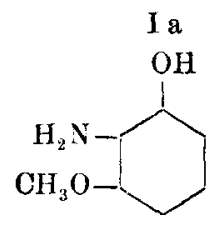<smiles>COC1CCC(N)C(O)C1</smiles><smiles>COC1CC(O)CCC1N</smiles>

Von diesen geht IIIa durch Oxydation mit Chromsäure leicht in Methoxychinon über, die beiden anderen lassen sich nicht so leicht zu einem Parachinon oxydieren.

Denkt man sich nun in diesen drei Reduktionsprodukten die Methoxylgruppe durch die Hydroxylgruppe versetzt:

1) Ber. 35, $147 \overline{5}$. 
Henrich u. Eisenach: Finw. von salp. Säure etc. 333
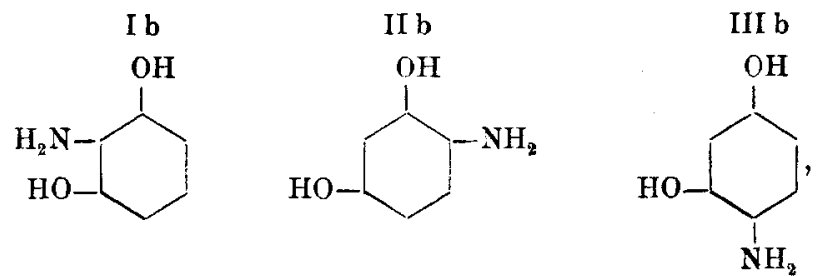

so sieht man, daB aus II a und III a ein und dasselbe A midoorcin entstehen muB, aus $I$ a aber ein davon verschiedenes.

Um diesen Ersatz der Methoxyl- durch die Hydroxylgruppe durchzuführen, wurde je $1 \mathrm{~g}$ des salzsauren Amidophenoläthers mit $10 \mathrm{~g}$ konzentrierter Salzsäure eine Stunde lang im Rohr auf $150^{\circ}$ erhitzt. Nach dem Erkalten und Öffnen des Rohres entwich ein Gas, das mit grün gesäumter, leuchtender Flamme brannte und offenbar Chlormethyl war. Der Rohrinhalt wurde im Vakuum auf ein kleines Volumen eingeengt und zur Kristallisation gestellt.

Es ergab sich nun die bemerkenswerte Tatsache, daB aus allen drei Reduktionsprodukten ein und dasselbe Chlorhydrat auskristallisierte. Dies wurde auch dadurch erwiesen, daB die aus den drei verschiedenen Versuchen enthaltenen Chlorhydrate sich vermittelst Essigsäureanhydrid in ein- und dasselbe Acetylderivat überführen ließen. Das salzsaure Amidoresorcin aber, welches sich durch Verseifung der drei Reduktionsprodukte gebildet hatte, war identisch mit dem 4-Amidoresorcin<smiles>NC1CCC(O)CC1O</smiles>

dessen Konstitution durch die Untersuchung des einen von uns mit B. Wagner festgestellt ist. ${ }^{1}$ ),

$\mathrm{Da}$ bei den eingehaltenen Reaktionsbedingungen eine Umlagerung der Amidogruppe von einem Ort im Benzolkern zu einem anderen wohl ausgeschlossen ist, so müBten auch die zwei von Henrich und Rhodius erhaltenen Ortho-

1) Ber. 35, 4195 (1902). 
334 Henrich u. Eisenach: Einw. von salp. Säure etc. nitrosoresorcinmonomethyläther die Nitrosogruppe an ein und derselben Stelle im Molekül enthalten und beide eine Konstitution entsprechend Formel II besitzen. Da nun von diesen Ortho-Nitrosoäthern einer bereits in zwei Modifikationen erhalten wurde, so müBten bei der Substituentenverteilung in Formel II drei Isomere existieren, was sich theoretisch nicht erklären ließe.

Um hier Klarheit zu schaffen, haben wir die Produkte von Rhodius von neuem untersucht. Ein Reservepräparat, das noch Rhodius dargestellt hatte, zeigte in der Tat ein ganz anderes Aussehen wie der isomerisierbare Äther. Während letzterer aus Benzol-Ligrö̈n in intensiv dunkelgrün gefärbten Blättern kristallisierte, kam der erstere aus dem gleichen Lösungsmittel in grau glänzenden, abgerundeten Kristallen, die auch beim öfteren Umkristallisieren ihre Farbe nicht änderten. Als wir den Schmelzpunkt des von Rhodius dargestellten Präparates nahmen, fanden wir in Übereinstimmung mit den a. a. O. gemachten Angaben bei $138^{\circ}$ eine starke Sinterung, indessen kam die Masse nicht zum völligen Schmelzen, sondern blieb im halb geschmolzenen Zustand und begann allmählich, wenn auch schwach, ihre Farbe zu verändern. Völlige Zusammenschmelzung fand erst bei $154^{\circ}$ statt. Durch diese Beobachtung war es wahrscheinlich geworden, dab der von Rhodius ${ }^{1}$ ) und dem einen von uns beschriebene $\beta$-Nitrosoresorcinmonomethyläther nur unreiner, isomerisierter $\alpha$-Nitrosoresorcinmonomethyläther ist. Diese Vermutung wurde zur Sicherheit, als jenes noch von Rhodins herrührende Präparat nach längerem Liegen erst an einzelnen Stellen, dann mehr und mehr gelb wurde und nunmehr ohne vorherige Sinterung und Farbenänderung den Schmelzp. $154^{\circ}$ zeigte. Der zweite von Rhodius und $\mathrm{mir}^{2}$ ) beschriebene sogenannte $\beta$-Nitrosoresorcinmonomethyläther ist somit nichts anderes, als isomerisierter $\alpha$-Ortho-Nitrosoresorcinmonomethyläther, der - wohl infolge kleiner Verunreinigungen - keine grüne Farbe zeigte. ${ }^{3}$ )

1) Ber. 35, 1484.

2) Das.

3) Anm. Kietaibls $\beta$-Ortho-Nitrosoresorcinmonoäthyläther vom Schmelzp. $101^{\circ}$ (vergl. Monatshefte 19,548) habe ich bisher nie in ganz reinem Zustande erhalten können. Des öfteren entstanden beim Um. 
Henrich u. Eisenach: Einw. von salp. Säure etc. 335

Bei der Einwirkung von salpetriger Säure auf Resorcinmonomethyläther entstehen somit nur zwei ortsisomere Mononitrosoderivate, von denen sich im einen Fall die Nitroso. und die freie Hydroxylgruppe in Ortho-, im anderen in Parastellung befinden. Die Substituentenverteilung der beiden Nitrosoresorcinmonomethyläther wird durch die Formeln II und III ausgedrückt.

Nun zeigte ich in Gemeinschaft mit Herrn Rhodius, daß der Orthonitrosoäther (II) in zwei ähnlichen Modifikationen existiert, wie Nitrosoresorcinmono äthyläther (s. die vorvorkergehende Abhandlung). Die labile Modifikation ist grün, die stabile braungelb gefärbt.

Herr Professor L enk war so liebenswürdig, diese beiden Modifikationen auf ihre kristallographischen Eigenschaften hin zu untersuchen, soweit das bei der Unvollkommenheit der Kristalle möglich war. Auch an dieser Stelle danke ich Herrn Professor Lenk verbindlichst für seine Bemühung. Wie beim Nitrosoresorcinmonoäthyläther ist auch hier die grüne, labile Modifikation stark dichroitisch. Die lauchgrünen Kristalle des Methyläthers gehören wahrscheinlich dem rhombischen Systeme an. Die gelbe, stabile Modifikation ist nicht dichroitisch und kristallisiert prismatisch.

Erhitzt man die ganzen grünen Kristalle im Schmelzpunktsröhrchen, so wandeln sich die reinsten Präparate je nach der Schnelligkeit des Erhitzens bei $130^{\circ}-140^{\circ}$ ziemlich rasch in die gelben um und schmelzen beim weiteren Erhitzen bei $158^{\circ}-159^{\circ}$. Auch hier findet die Umwandlung bei gewöhnlicher Temperatur allmählich statt. Beim längeren Liegen beginnen in den grünen Kristallen gelbe Punkte und Streifen aufzutreten, die sich vergrößern, bis schließlich der

kristallisieren des in Benzol leichter Löslichen aus Alkohol Produkte, die anders aussahen als der $\alpha$-Äther und auch niedriger schmolzen. Sie konnten durch öfteres Umkristallisieren zwar auf den höheren Schmelzpunkt gebracht werden, aber erst nach der Verwandlung in das Kaliumsalz, Wiederabscheidung durch Säuren und Umkristallisieren aus Alkobol entstanden die goldgelben Blättchen, die für den $\alpha$-Äther so charakteristisch sind. Durch Einengen der Benzolmutterlaugen entstanden neue Kristallisationen, die aber immer in $\alpha$-Äther übergeführt werden konnten. Wenn der Ortho-Äther vom Schmelzp. $101^{\circ}$ wirklich existier, dann ist er jedenfalls nicht so leicht zu erhalten, wie Kietaibl angibt. 
336 Henrich u. Eisenach: Einw. von salp. Säure etc.

ganze Kristall gelb ist. Indessen ist die grüne Modifikation des Nitrosoresorcinmonomethyläthers wesentlich beständiger, als die des entsprechenden Äthyläthers und es davert längere Zeit, bis die spontane Umwandlung vollendet ist.

Das Verhalten gegen Lösungsmittel ist auch hier ähnlich wie bei dem entsprechenden Äthyläther. Aus assoziierenden Lösungsmitteln, besonders aus Benzol, Toluol und Schwefelkohlenstoff kristallisiert die grüne Modifikation, dissoziierend wirkende Solventien scheiden die gelbe aus. Versetzt man eine Benzollösung, die beim Abkühlen stets die labile, grüne Modifikation ausscheidet, mit mehr und mehr Alkohol, so kommt nach Zusatz weniger Tropfen ein Punkt, wo sich nicht mehr die grüne, sondern die gelbe Modifikation ausscheidet. Während aber bei dem entsprechenden Nitrosoresorcinmonoäthyläther der Zusatz eines Tropfens zu mehreren Kubikzentimetern Benzollösung genügte, um diese Umwandlung zu bewirken, sind beim Methyläther - der gröBeren Beständigkeit seiner grünen Modifikation entsprechend - ungefähr ebenso viele Tropfen Alkohol nötig, als Kubikzentimeter Benzol-Lösung vorhanden sind.

Fine Molekulargewichtsbestimmung in alkoholischer Lösung hat Herr Rhodius ${ }^{1}$ ) bereits ausgeführt und hier die einfache Molekulargröße gefunden. Auch in einer Lösung von reinen Benzol, welche die grüne Modifikation enthält, fanden wir das einfache Molekulargewicht. $0,187^{\circ}$.

$0,2184 \mathrm{~g}$ Substanz erhöhten den Siedepunkt von $17,8 \mathrm{~g}$ Benzol um $0,297^{\circ}$.

$0,3438 \mathrm{~g}$ Substanz erlöhten den Siedepunkt von $17,8 \mathrm{~g}$ Benzol um

$0,4598 \mathrm{~g}$ Substanz erböhten den Siedepunkt von $17,8 \mathrm{~g}$ Benzol um $0,382^{\circ}$.

$$
\begin{array}{cccc}
\text { Berechnet: } & \multicolumn{3}{c}{\text { Gefunden: }} \\
M=153 & 171 & 169 & 176 .
\end{array}
$$

Nachdem es nunmehr feststeht, daB die beiden Modifikationen die gleiche chemische Zusammensetzung, Konstitution und Molekulargröße besitzen, erhebt sich die Frage, durch welche Ursachen die Verschiedenheit bedingt ist. Zwei Annahmen sind möglich, nämlich Polymorphie und Desmotropie.

1) Ber. 35, 1478. 
Henrich u. Eisenach: Einw. von salp. Säure etc. 337

Beim Erhitzen auf $130^{\circ}-140^{\circ}$ geht die grüne labile Modifikation in die gelbe stabile über, ohne daß der feste Aggregatzustand verlassen wird. Den umgekehrten Vorgang kann man indessen nicht so einfach vollziehen. Man muß vielmehr erst die stabile Modifikation in assoziierenden Lösungsmitteln auflösen, also den festen Aggregatzustand aufgeben, um die labile Modifikation wieder zu erzeugen. Würde also die Verschiedenheit beider Modifikationen auf Polymorphie beruhen, so läge ein Fall von Monotropie vor. Da mit dieser Annahme aber die Forderungen nicht in Einklang stehen, welche die physikalische Chemie aus guten Gründen für diesen Fall von Polymorphie aufgestellt hat, so bleibt nur die Annahme übrig, daB die beiden Modifikationen des Mononitrosoresorcinmonomethyläthers einen Fall von Desmotropie vorstellen.

Zunächst spricht für Desmotropie schon die Tatsache, daß man ähnliche Modifikationen bereits mehrfach gerade bei Nitrosoverbindungen beobachtet hat. ${ }^{1}$ ) Dann erinnert die Tatsache, daB sich der Nitrosoäther mit grüner Farbe in gewissen Lösungsmitteln löst, sehr an das ganz allgemeine Verhalten von Körpern, die eine wahre Nitrosogruppe besitzen. Der grünen Modifikation wäre somit die wahre Nitrosoformel, der gelben die Konstitution des entsprechenden Chinonoxims zu erteilen:<smiles>COc1ccc(C#N)c(O)c1</smiles>

grüne Blätter, die sich bei $130^{\circ}$ bis $140^{\circ}$ in die gelbe Modifikation umwandeln, dichroitisch.

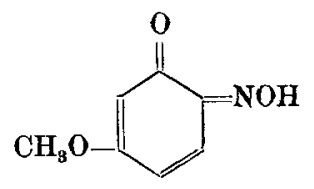

braungelbe bis gelbe Kristalle vom Schmelzp. 1680, nicht dichroitisch.

Chemische Unterschiede, besonders bei Lösungen in Benzol und Alkohol, konnten bis auf die unten beschriebene Reaktion mit Phenylcyanat bisher nicht festgestellt werden. Offenbar findet die Umwandlung der grünen Modifikation in die gelbe besonders in Lösungen $\mathrm{zu}$ leicht statt. Das beweist der folgende Versuch.

1) Vergl. F. Kehrmann, Ber. 39, 1415;31, 2413, 2117; 33, 1539, sowie die vorvorhergehende Abhandlung.

Journal t. prakt. Chemio [2] BA. 70 . 
338 Henrich u. Eisenach: Einw. von salp. Säure etc.

Wenn in Benzollösung vorzugsweise nur die grüne, in alkoholischer die gelbe Modifikation existiert, dann müßten beide Lösungen verschieden mit Phenylcyanat reagieren, wenn obiger Desmotropiefall vorläge, denn im einen Fall sitzt die Hydroxylgruppe am Kohlenstoff, im anderen am Stickstoff:

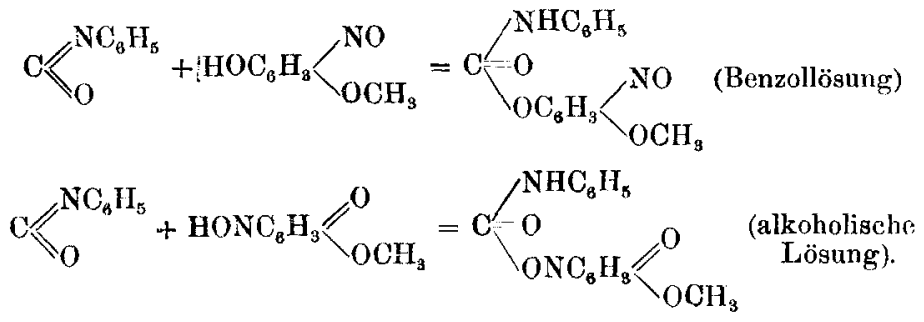

LäBt man die Reaktion in einer Lösung von 1 Teil Substanz auf 20 Teilen Lösungsmittel bei gewöhnlicher Temperatur vor sich gehen, so entsteht ein und dasselbe Urethan mit gleicher Geschwindigkeit. Bei größerer Verdünnung und niedrigerer Temperatur entsteht aus beiden Lösungen zwar ein und dasselbe Urethan, allein die Benzollösung scheidet letzteres erst nach viel längerer Zeit aus als die alkoholische Reaktionsflüssigkeit. Bei folgender Versuchsanordnung trat diese Erscheinung sehr deutlich hervor.

Je $0,4 \mathrm{~g}$ Nitrosoresorcinmonomethyläther wurden einerseits in $4 \mathrm{ccm}$ Benzol, andererseits in $4 \mathrm{ccm}$ Alkohol (oder Aceton) gelöst, die Lösungen auf $7^{\circ}$ abgekühlt und mit je $0,3 \mathrm{~g}$ Phenylcyanat versetzt. Die alkoholische und Acetonlösung schieden schon nach wenigen Sekunden ein Reaktionsprodukt aus, während es bei der Benzollösung etwa eine Viertelstunde dauerte, bis die ersten Kristallansätze begannen. Die Reaktionsprodukte wurden mehrmals unmittelbar nach der Abscheidung abgesaugt, getrocknet und auf den Schmelzpunkt hin untersucht. Stets begannen beide Produkte bei $162^{\circ} \mathrm{zu}$ sintern und schmolzen bei $168^{\circ}$. Die Elementaranalyse stimmte auf das erwartete Urethan.

$0,147 \mathrm{~g}$ Substanz gaben $0,3345 \mathrm{~g} \mathrm{CO}_{2}$ und $0,057 \mathrm{~g} \mathrm{H}_{2} \mathrm{O}$.

$0,2112 \mathrm{~g}$ Substanz gaben $19,6 \mathrm{~cm} \mathrm{~N}$ bei $22^{\circ}$ und $755 \mathrm{~mm}$.

Berechnet für $\mathrm{C}_{14} \mathrm{H}_{12} \mathrm{O}_{4} \mathrm{~N}_{2}$ :

Gefunden:

$$
\begin{aligned}
& \mathrm{C}=61,8 \\
& \mathrm{H}=4,4 \\
& \mathrm{~N}=10,3
\end{aligned}
$$$$
4,35,
$$$$
10,6 " \text {. }
$$ 
Henrich u. Eisenach: Einw. von salp. Säure otc. 339

Das Urethan des Nitrosoresorcinmonomethyläthers ist in den üblichen organischen Lösungsmitteln durchweg sehr schwer löslich. Beim Kochen mit Alkohol wird das Phenylcyanat wieder abgespalten.

Die Tatsache, daß man aus der Lösung des Nitrosoäthers in Benzol einerseits und Alkohol oder Aceton andererseits zwar ein und dasselbe Urethan, aber mit verschiedener Geschwindigkeit erhält, läßt sich meines Erachtens auch am besten mit der Annahme erklären, daB die beiden Modifikationen im Sinne der Formeln auf S. 337 desmotrop sind. Das Phenylcyanat lagert eben in der Benzollösung zuerst das wahre Nitrosophenol in das Chinonoxim um, diese Umlagerung erfordert Zeit und darum die spätere Abscheidung des Urethans aus der Benzollösung. In der Tat verhält sich Phenylcyanat wie ein dissoziierendes Lösungsmittel, denn es löst vor der Urethanbildung den festen Nitrosoäther mit ähnlich gelber Farbe auf wie Alkohol. Genau so wie letzterer beim Zusatz zur Benzollösung die Abscheidung der grünen Modifikation zu verhindern vermag und die Umlagerung in die gelbe Modifikation bewirkt, ist auch Phenylcyanat hierzu imstande. Und erst die umgelagerte gelbe Modifikation, also das Chinonoxim reagiert unter Urethanbildung mit dem Nitrosoäther. Dem Urethan ist aus diesen Gründen folgende Formel:

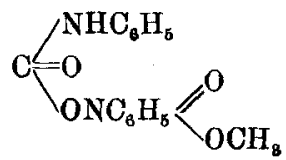

zu erteilen. In alkoholischer Lösung liegen aber die Moleküle gleich in umgelagerter Form vor, und darum kann die Urethanbildung sofort vor sich gehen.

Gibt man zu Lösungen des Nitrosoresorcinmonomethyl-

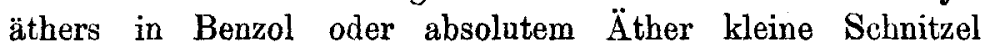
metallischen Natriums, so entsteht aus beiden Lösungen ein und dasselbe Natriumsalz, und wie bisher auch die Reaktionsbedingungen gestaltet wurden, stets lieferten beide Modifikationen ein und dasselbe Alkalisalz.

Die Lösung der Alkalisalze reagiert neutral. Säuert man die wäßrige Lösung dieser Alkalisalze in der Hitze oder in der Kälte an, oder leitet man einen starken elektrischen Strom 
340 Henrich u. Eisenach: Einw. von salp. Säure etc.

durch ihre sehr verdünnte Lösung, so scheidet sich nie die grüne, sondern stets die gelbe Modifikation ab. Schon diese Tatsache macht es wahrscheinlich, daß sich die Alkalisalze von der gelben Modifikation, also von Chinonoxim ableiten. Als eine $1 / 100$ n-wäBrige Lösung des Kaliumsalzes mit der äquivalenten Menge $1 / 100$ n-Salzsäure versetzt wurde, lieB sich ein Dissoziationsrückgang durch die Bestimmung der Leitfähigkeit nicht beobachten.

Endlich wurde der Dimethyläther des Nitrosoresorcins dargestellt. Versuche, das Kaliumsalz mit Jodmethyl umzusetzen, führten zu so unbefriedigenden Resultaten, daß wir das Silbersalz zu Hülfe nehmen mußten. Man kann letzteres leicht gewinnen, wenn man äquivalente Mengen von Kaliumsalz und Silbernitrat in wäßriger Lösung auf einander einwirken läBt. Das ausfallende braune Silbersalz wurde gewaschen, scharf getrocknet und fein gepulvert mit einer ätherischen Lösung von Jodmethyl in Reaktion gebracht. Die Umsetzung geht leicht vor sich und beim Verdunsten der filtrierten ätherischen Lösung entsteht eine Masse, die sich durch öfteres Umkristallisieren aus Ligroïn in schöne hellgelbe Prismen vom Schmelzp. $115^{\circ}-117^{\circ}$ verwandeln lieB. Die Analyse stimmte auf einen Dimethyläther.

$0,1698 \mathrm{~g}$ Substanz gaben $0,3567 \mathrm{~g} \mathrm{CO}_{2}$ und $0,0857 \mathrm{~g} \mathrm{H}_{2} \mathrm{O}$.

$0,1135 \mathrm{~g}$ Substanz gaben $8,7 \mathrm{ccm} \mathrm{N}$ bei $21^{\circ}$ und $735 \mathrm{~mm}$.

Berechnet für $\mathrm{C}_{8} \mathrm{H}_{9} \mathrm{O}_{8} \mathrm{~N}$ :

Gefunden:

$$
\begin{aligned}
& \mathbf{C}=57,5 \\
& \mathrm{H}=5,4 \\
& \mathrm{~N}=8,4
\end{aligned}
$$

$57,2 \%$
$5,66 "$
$8,46 "$.

In Alkohol, Benzol und Eisessig ist der Dimethyläther momentan löslich. Äther löst in der Kälte eine mäBige Menge, in der Wärme mehr auf. Von Ligrö̈n wird in der Kälte wenig, bei Siedetemperatur mehr aufgenommen. Kalte wäBrige Soda und Alkalilauge lassen die Verbindung unverändert.

Schon die Tatsache, daß der Dimethyläther gelb gefärbt ist und sich mit gelber und nicht mit grüner Farbe in organischen Lösungsmitteln löst, weist darauf hin, daß er kein echter Nitrosokörper ist. Reduziert man ihn mit Zinnchlorür und Salzsäure, so entsteht ein Chlorhydrat, das bei der 
Henrich a. Eisenach: Einw. von salp. Säure etc. 341 Acetylierung in ein Triacetylderivat vom Schmelzp. 92 $^{\circ}$ übergeht. Dasselbe Triacetylderivat haben Rhodius und der eine von uns bereits früher ${ }^{1}$ ) aus dem Nitrosoresorcin mo no methyläther erhalten. Eine Elementaranalyse bestätigte die Identität beider Verbindungen.

$0,16 \mathrm{~g}$ Substanz gaben $0,344 \mathrm{~g} \mathrm{CO}_{2}$ und $0,085 \mathrm{~g} \mathrm{H}_{2} \mathrm{O}$.

$\begin{array}{cc}\text { Berechnet: } & \text { Gefunden: } \\ \mathbf{C}=58,8 & \mathbf{5 8 , 6 4} \% \\ \mathrm{H}=5,7 & 5,95 \%\end{array}$

Dem Dimethyläther dürfte somit die Formel:

zukommen.<smiles>CON=C1C=CC(OC)=CC1=O</smiles>

1) Ber. 35, 1480. 